CU-TP-539

FERMILAB-Pub-91/312-A\&T

\title{
Black Holes in Magnetic Monopoles
}

\author{
Kimyeong Lee, ${ }^{*}$ V.P.Nair \\ Physics Department, Columbia University \\ New York, New York 10027 \\ and \\ Erick J. Weinberg \\ Physics Department, Columbia University \\ New York, New York 10027 \\ and \\ Theory Group and NASA/Fermilab Astrophysics Center \\ Fermi National Accelerator Laboratory \\ P.O.Box 500, Batavia, Illinois 60510.
}

\begin{abstract}
We study magnetically charged classical solutions of a spontaneously broken gauge theory interacting with gravity. We show that nonsingular monopole solutions exist only if the Higgs field vacuum expectation value $v$ is less than or equal to a critical value $v_{c r}$, which is of the order of the Planck mass. In the limiting case, the monopole becomes a black hole, with the region outside the horizon described by the critical Reissner-Nordstrom solution. For $v<v_{c r}$, we find additional solutions which are singular at $r=0$, but which have this singularity hidden within a horizon. These have nontrivial matter fields outside the horizon, and may be interpreted as small black holes lying within a magnetic monopole. The nature of these solutions as a function of $v$ and of the total mass $M$ and their relation to the Reissner-Nordstrom solutions is discussed.
\end{abstract}

This work was supported in part by the US Department of Energy (VPN and EJW), by NASA (EJW) under grant NAGW-2381 and by an NSF Presidential Young Investigator Award (KL).

* Alfred P. Sloan Fellow. 


\section{Introduction}

Some spontaneously broken gauge theories contain magnetic monopoles which have the remarkable property that, despite being particles in a quantum theory, they are described by a classical field configuration. This is possible because in the limit of weak gauge coupling $e$ their Compton wavelength $\sim e / v$ is much less than the radius $\sim 1 /(e v)$ of the classical monopole solution. Another curious property emerges as the Higgs vacuum expectation value $v$ approaches the Planck mass $M_{P}$. The Schwarzschild radius $2 M G \sim v /\left(e M_{P}^{2}\right)$ becomes comparable to the monopole radius, suggesting that for $v \gtrsim M_{P}$ the monopole should be a black hole. ${ }^{[1]}$ (This result can be evaded in theories containing dilatons. $\left.{ }^{[2]}\right)$ If $e \ll 1$, this occurs in a regime where the energy density is much less than $M_{P}$, justifying the neglect of quantum gravity effects. By studying the classical solution, then, one can gain insight into how the particle passes over into a black hole.

In this paper we undertake such an investigation. We consider an $S U(2)$ gauge theory in which a triplet Higgs field $\phi$ breaks the symmetry down to $U(1) ;{ }^{*}$ this theory gives rise to 't Hooft-Polyakov monopoles with magnetic charge $Q_{M}=1 / e$. We find that when $v=\langle\phi\rangle$ is sufficiently large, the only magnetically charged solutions are the Reissner-Nordstrom black holes. These are essentially Abelian, in that the only nontrivial matter field is the Coulomb magnetic field lying in the unbroken $U(1)$ subgroup. They have a singularity at $r=0$ whose strength is determined by the mass $M$. In order that this singularity be hidden within a horizon, $M$ must be greater than $M_{c r i t}=\sqrt{4 \pi Q_{M}^{2}} M_{P}$. For smaller values of $v$, however, we find that a rather different type of black hole solutions is also possible. For these the horizon lies within the core of the monopole, so that the non-Abelian structure is quite evident in the region outside the horizon. In a sense, these solutions can be viewed as black holes lying inside monopoles. The mass of these objects can take any value down to the mass of the nonsingular monopole. We find that there is also an upper limit on their mass. In some cases this limit is

\footnotetext{
* There has been some study ${ }^{[3]}$ of black hole like solutions in the theory without Higgs fields.
} 
greater than the critical Reissner-Nordstrom mass, so that there are two different black hole solutions with the same values for the mass and magnetic charge.

Classical solutions can also play another role in relation to semiclassical gravity. Black holes can reduce their mass by Hawking radiation. By this mechanism initially macroscopic black holes can shrink to the microscopic size characteristic of the classical solutions. (In the weak coupling limit this scale is much greater than the Planck length, so gravity can still be treated semiclassically.) Once this happens, the classical solutions provide possible pathways for the further evolution of the black hole by the Hawking process. An understanding of the nature of these solutions as a function of coupling constants and other parameters can thus lead to further insight into the late stages of magnetically charged black holes.

In Sec. 2 we review the essential features of the theory and obtain the field equations which must be obeyed by static spherically symmetric solutions. Much of this reproduces, although with a somewhat different notation, the results of van Nieuwenhuizen, Wilkinson, and Perry ${ }^{[4]}$. In Sec. 3 we study nonsingular monopole solutions and their behavior as $v$ approaches $M_{P}$. In Sec. 4 we extend our considerations to include solutions with singularities inside the horizon which are essentially black holes inside the monopole. Section 5 contains some concluding remarks. There are two appendices. In the first, we reconcile the absence of nonsingular solutions for large $v$ with the existence of a positive definite functional whose minima are solutions of the field equations. In the second, we derive a number of inequalities which restrict the properties of the various types of black hole solutions. 


\section{General Formalism}

The theory is governed by the action

$$
S=\int d^{4} x \sqrt{-g}\left[-\frac{1}{16 \pi G} R+\mathcal{L}_{\text {matter }}\right]
$$

where

$$
\mathcal{L}_{\text {matter }}=-\frac{1}{4}\left(F_{\mu \nu}^{a}\right)^{2}+\frac{1}{2}\left(D_{\mu} \phi^{a}\right)^{2}-\frac{\lambda}{2}\left(\left(\phi^{a}\right)^{2}-v^{2}\right)^{2}
$$

with Latin indices $a, b, \ldots$ referring to the internal $S U(2)$ indices,

$$
\left(D_{\mu} \phi\right)^{a}=\partial_{\mu} \phi^{a}-e \epsilon_{a b c} A_{\mu}^{b} \phi^{c}
$$

and

$$
F_{\mu \nu}^{a}=\partial_{\mu} A_{\nu}^{a}-\partial_{\nu} A_{\mu}^{a}-e \epsilon_{a b c} A_{\mu}^{b} A_{\nu}^{c}
$$

A constant term has been included in the scalar field potential so that the energy vanishes in the symmetry-breaking vacuum. The elementary excitations about this vacuum are a massless photon, two charged vectors with mass $m_{V}=e v$, and a neutral massive Higgs scalar with mass $m_{H}=2 \sqrt{\lambda} v$.

In this paper we consider only static spherically symmetric solutions. For these, the metric may be written in the form

$$
d s^{2}=B(r) d t^{2}-A(r) d r^{2}-r^{2}\left(d \theta^{2}+\sin ^{2} \theta d \phi^{2}\right)
$$

The normalization of $t$ is fixed by requiring that $B(\infty)=1$, while the requirement that space be asymptotically flat imposes the condition $A(\infty)=1$. For later convenience we define $\mathcal{M}(r)$ by

$$
A(r)=\left[1-\frac{2 G \mathcal{M}(r)}{r}\right]^{-1}
$$

For the matter fields we adopt the standard spherically symmetric ansatz corresponding to magnetic charge $Q_{M}=1 / e$. In flat space this ansatz is usually 
written in terms of Cartesian coordinates as

$$
\begin{gathered}
\phi^{a}=v \hat{r}^{a} h(r) \\
A_{i}^{a}=\epsilon_{i a k} \hat{r}^{k} \frac{1-u(r)}{e r} \\
A_{0}=0
\end{gathered}
$$

The extension to curved space ${ }^{[4]}$ is most easily done by first transforming to spherical coordinates. ${ }^{*}$ Once this has been done, the matter part of the action can be written as

$$
S_{\text {matter }}=-4 \pi \int d t d r r^{2} \sqrt{A B}\left[\frac{K(u, h)}{A}+U(u, h)\right]
$$

where

$$
K=\frac{u^{\prime 2}}{e^{2} r^{2}}+\frac{1}{2} v^{2} h^{\prime 2}
$$

and

$$
U=\frac{\left(u^{2}-1\right)^{2}}{2 e^{2} r^{4}}+\frac{u^{2} h^{2} v^{2}}{r^{2}}+\frac{\lambda}{2} v^{4}\left(h^{2}-1\right)^{2}
$$

(Primes denote differentiation with respect to $r$.)

$U(u, h)$ may be viewed as a position-dependent field potential. For later reference, we enumerate here its stationary points:

a) $u= \pm 1, h=0$ : This is a local minimum of $U$ if $r<1 /(\sqrt{\lambda} v)$, and is a saddle point otherwise.

b) $u=0, h=0$ : This is always a local maximum of $U$.

* An invariant way to write the ansatz for the vector potential is

$$
A_{\mu}^{a} d x^{\mu}=f L^{a \mu} g_{\mu \nu} d x^{\nu}
$$

where $L^{a \mu} \frac{\partial}{\partial x^{\mu}}$ are the three Killing vectors corresponding to the rotational symmetry and $f$ is a function invariant under the action of these Killing vectors. For the choice of spherical coordinates in Eq. (2.5), this is the same as Eqs. (2.8) and (2.9). 
c) $u=\hat{u}(r), h=\hat{h}(r)$, where

$$
\begin{aligned}
& \hat{u}(r)=\sqrt{\frac{\lambda\left(1-e^{2} r^{2} v^{2}\right)}{\lambda-e^{2}}} \\
& \hat{h}(r)=\sqrt{\frac{\lambda r^{2} v^{2}-1}{\left(\lambda-e^{2}\right) r^{2} v^{2}}}
\end{aligned}
$$

These are both real only when $r v$ lies between $1 /|e|$ and $1 / \sqrt{\lambda}$. Within this range of $r$, this point is the global minimum of $U$ if $\lambda>e^{2}$, and a saddle point otherwise. When $\lambda=e^{2}, \hat{u}$ and $\hat{h}$ are undefined, and this stationary point is replaced by a degenerate set of minima, with $h^{2}+u^{2}=1$, which exist only when $|e| v r=1$.

d) $u=0, h= \pm 1$ : This is a local minimum of $U$ if $r>1 /(e v)$, but only a saddle point otherwise.

The gravitational field equations reduce to two independent equations, which may be written as

$$
\frac{(A B)^{\prime}}{A B}=16 \pi G r K
$$

and

$$
\mathcal{M}^{\prime}=4 \pi r^{2}\left(\frac{K}{A}+U\right)=4 \pi r^{2}(K+U)-8 \pi G r K \mathcal{M}
$$

The equations for the matter fields are

$$
\begin{aligned}
\frac{1}{\sqrt{A B}}\left(\frac{\sqrt{A B} u^{\prime}}{A}\right)^{\prime} & =e^{2} \frac{r^{2}}{2} \frac{\partial U}{\partial u} \\
& =\frac{u\left(u^{2}-1\right)}{r^{2}}+e^{2} u h^{2} v^{2}
\end{aligned}
$$

and

$$
\begin{aligned}
\frac{1}{r^{2} \sqrt{A B}}\left(\frac{r^{2} \sqrt{A B} h^{\prime}}{A}\right)^{\prime} & =\frac{1}{v^{2}} \frac{\partial U}{\partial h} \\
& =\frac{2 h u^{2}}{r^{2}}+2 \lambda h\left(h^{2}-1\right) v^{2}
\end{aligned}
$$

By rescaling of distances in these equations, it can be shown that that $u, h$ and 
$e \mathcal{M} / v$ are given by functions of $e v r$ whose forms depend only on $\lambda / e^{2}$ and $G v^{2}=$ $\left(v / M_{P}\right)^{2}$.

By integration of (2.16) we see that the mass can be written as

$$
M \equiv \mathcal{M}(\infty)=4 \pi \int_{0}^{\infty} d r r^{2} e^{-P(r)}(K+U)+e^{-P(0)} \mathcal{M}(0)
$$

where

$$
P(r)=\int_{r}^{\infty} d r 8 \pi G r K
$$

If $\mathcal{M}(0) \geq 0$, the mass defined by (2.19) satisfies the inequality ${ }^{[4]}$

$$
M \geq e^{-P(0)}\left[4 \pi \int_{0}^{\infty} d r r^{2}(K+U)+\mathcal{M}(0)\right] \geq \frac{4 \pi v}{e} e^{-P(0)}
$$

which follows from the positivity of $K$ and the Bogomol'nyi bound. ${ }^{[5]}$ For a nonsingular solution, $\mathcal{M}(0)=0$. A solution $u, h$ is then a minimum of $M$, viewed as a functional of $u$ and $h$, and so we have

$$
M(u, h) \leq M\left(u_{0}, h_{0}\right) \leq\left. 4 \pi \int_{0}^{\infty} d r r^{2}(K+U)\right|_{u_{0}, h_{0}} \leq M_{\text {flat }}
$$

where $u_{0}$ and $h_{0}$ are the flat-space solutions and $M_{\text {flat }}$ is the flat-space monopole mass. This inequality is of course in accord with our intuition that gravity tends to reduce the mass.

Because the function space is noncompact, there is no guarantee that there will actually be a configuration which minimizes $M$. Instead, there could be an infinite sequence of configurations of decreasing energy which does not converge on a limiting configuration. Indeed, for $G v^{2}$ sufficiently large we find that there are no nonsingular solutions. In Appendix A we display a sequence of configurations for which $M$ approaches, but does not reach, its lower bound for this case. 
Eq. (2.15) can be used to eliminate $B(r)$ from the remaining field equations, leaving one first-order and two second-order equations to be integrated. A solution of these is determined by five boundary conditions. Two are provided by the asymptotic conditions $u(\infty)=0$ and $h(\infty)=1$. The remaining three can be obtained by requiring that the solution be nonsingular at the origin, which implies that $u(0)=1, h(0)=0$, and $\mathcal{M}(0)=0$.

Matters become more complicated if horizons are present, i.e., if $1 / A(r)$ has zeros. At a horizon $r_{H}$, the vanishing of $1 / A$ gives

$$
\mathcal{M}\left(r_{H}\right)=\frac{r_{H}}{2 G}
$$

This, together with Eq. (2.16), implies that

$$
\left(\frac{1}{A}\right)^{\prime}=\frac{1}{r}-8 \pi \operatorname{Gr} U(u, h), \quad r=r_{H}
$$

Substitution of this into Eqs. (2.17) and (2.18) gives two conditions

$$
u^{\prime}\left[\frac{1}{r}-8 \pi G U(u, h)\right]=\frac{e^{2} r^{2}}{2} \frac{\partial U}{\partial u}, \quad r=r_{H}
$$

and

$$
h^{\prime}\left[\frac{1}{r}-8 \pi G U(u, h)\right]=\frac{1}{v^{2}} \frac{\partial U}{\partial h}, \quad r=r_{H}
$$

which must hold if the solution is to be nonsingular.

Since these additional conditions overdetermine the solution, we do not expect there to be any nonsingular solutions containing horizons, except perhaps for special values of $\lambda$ and $v$. If singularities are allowed at $r=0$, then it should be possible to obtain otherwise nonsingular solutions with a single horizon, but not (for generic values of $\lambda$ and $v$ ) with two or more. 
Finally, note that a solution ${ }^{[6]}$ of the field equations is obtained by taking constant fields $u(r)=0$ and $h(r)=1$, with

$$
\mathcal{M}(r)=M-\frac{2 \pi}{e^{2} r}
$$

where $M$ is arbitrary. This yields the Reissner-Nordstrom metric

$$
B(r)=A(r)^{-1}=1-\frac{2 M G}{r}+\frac{4 \pi G}{e^{2} r^{2}}
$$

which has a singularity at $r=0$. There are horizons at

$$
r_{ \pm}=M G \pm \sqrt{M^{2} G^{2}-\frac{4 \pi G}{e^{2}}}
$$

provided that $M$ is greater than the critical value $M_{c r i t}=\sqrt{4 \pi /\left(G e^{2}\right)}$.

\section{Nonsingular Monopoles}

For values of $v$ much smaller than the Planck mass, gravitational effects on the monopole are small and the nonsingular monopole solutions should be similar to their flat-space counterparts. On the other hand, as we have argued above, if $v \gg M_{P}$, the Schwarzschild radius would be greater than the size of the monopole, so the monopole must be a black hole. In this section we examine the transition between these two regimes.

Near the origin, a nonsingular solution must behave as

$$
\begin{gathered}
u(r)=1-C_{u} r^{2}+\cdots \\
h(r)=C_{h} r+\cdots \\
\mathcal{M}(r)=\frac{4 \pi}{3}\left(\frac{6 C_{u}^{2}}{e^{2}}+\frac{3}{2} C_{h}^{2} v^{2}+\frac{\lambda}{2} v^{4}\right) r^{3}
\end{gathered}
$$

where $C_{u}$ and $C_{h}$ are constants which must be chosen so that $u$ and $h$ approach the correct values as $r \rightarrow \infty$. In the absence of gravity, the possibility of making 
such a choice is ensured by the existence of a positive definite energy functional whose minimum is a solution of the static field equations. This argument can be extended to the case of weak gravity ${ }^{[4]}$ (see Eq. (2.19)), although, as discussed in Sec. 2 and Appendix A, it fails when $v / M_{P}$ becomes too great.

Just as in flat space, the matter fields $u$ and $h$ remain nontrivial inside the monopole "core" of radius $\sim 1 /(e v)$ and then approach their asymptotic values exponentially fast:

$$
\begin{gathered}
u(r)=O\left(e^{-m_{V} r}\right) \\
h(r)=1-O\left(e^{-m_{H} r}\right)
\end{gathered}
$$

It then follows from Eq. (2.16) that

$$
\mathcal{M}(r)=M-\frac{2 \pi}{e^{2} r}+O\left(e^{-m_{V} r}, e^{-m_{H} r}\right)
$$

In flat space, the monopole mass $M_{\text {mon }} \equiv M_{\text {flat }}=(4 \pi v / e) f\left(\lambda / e^{2}\right)$, where $f$ ranges from 1 to 1.787 as $\lambda$ ranges from 0 to $\infty .^{[7]}$ As mentioned above, gravitational effects cause $M$ to be somewhat smaller; our numerical results indicate that the monopole mass can be reduced to about two-thirds of its flat-space value.

We now turn to the discussion of how a horizon develops as the mass increases. From the large and small $r$ behavior of $\mathcal{M}$, it is evident that $1 / A$ will have a minimum, corresponding to a maximum of $\mathcal{M} / r$, at some intermediate value of $r$. The asymptotic form Eq. (3.6) suggests that this occurs at a value $\bar{r} \sim 4 \pi /\left(e^{2} M\right) \sim$ $1 /(e v)$, with $1 / A(\bar{r}) \approx 1-O\left(G v^{2}\right)$. As $v$ increases, this minimum should become deeper, until eventually a critical value $v_{c r}$ is reached for which $1 / A(\bar{r})=0$ and a horizon appears. One would expect this horizon to persist if $v$ were increased further, but, as was argued in the previous section, it will not in general be possible for a solution with proper asymptotic behavior to be well-behaved at both the horizon and at $r=0$. We therefore expect that only singular solutions exist when $v>v_{c r}$. More specifically, our results for the critical case suggest that in the 
supercritical case there are only Reissner-Nordstrom solutions with $u(r)=0$ and $h(r)=1$; in the next section we will prove this to be the case if $v$ is sufficiently great.

Let us examine the critical case $v=v_{c r}$ in more detail. To begin, note that Eqs. (2.25) and (2.26), together with the fact that $1 / A$ is stationary at the horizon, imply that $u\left(r_{H}\right)$ and $h\left(r_{H}\right)$ must correspond to one of the stationary points of $U(u, h)$, which were enumerated in Sec. 2 The first two, $u= \pm 1, h=0$ and $u=0$, $h=0$, are easily ruled out. For the former, one can show that if $1 / A$ and $(1 / A)^{\prime}$ both vanish, then $(1 / A)^{\prime \prime}$ must be negative, in contradiction with the assumption that $1 / A$ is at a minimum. In the latter case, it is easy to show that all solutions of Eq. (2.17) and (2.18) develop singularities as $r \rightarrow r_{H}$ if $(1 / A)^{\prime \prime}>0$.

We have not been able to completely eliminate the third case, $u=\hat{u}(r), h=$ $\hat{h}(r)$. There are however several constraints which the parameters must satisfy for a solution to exist. ${ }^{[8]}$ The condition $(1 / A)^{\prime}=0$ implies $4 \pi G\left(u^{2}+h^{2}\right)=1$ at the horizon. With the values of $\hat{u}(r), \hat{h}(r)$ from Eqs. (2.13) and (2.14), this leads to a quadratic equation for $r_{H}^{2}$. The requirements that $v r_{H}$ lie between $1 / e$ and $1 / \sqrt{\lambda}$, so that $\hat{u}$ and $\hat{h}$ are both real, and that $(1 / A)^{\prime \prime}>0$ eliminate one of the solutions of the quadratic equation and lead to the conditions

$$
\begin{array}{ll}
1+\sqrt{\frac{e^{2}}{\lambda}} \geq 8 \pi G v^{2} \geq 2, & \lambda<e^{2} \\
1+\sqrt{\frac{e^{2}}{\lambda}} \leq 8 \pi G v^{2} \leq 2, & \lambda>e^{2}
\end{array}
$$

In addition to these requirements, the solution in the region within the horizon must be such that $2 G \mathcal{M}\left(r_{H}\right)=r_{H}$; we do not know whether this can be done with $v$ in the range specified above. Furthermore, we have not addressed the question of whether these solutions are stable; this seems particularly doubtful for the case $\lambda<e^{2}$, where $\hat{h}, \hat{u}$ is not a minimum of $U(h, u)$.

Finally, we come to the case $u=0, h=1$. This corresponds to a solution in which $u$ and $h$ have already reached their asymptotic values at the horizon which, 
from Eq. (2.24), must occur at

$$
r_{H}=\sqrt{\frac{4 \pi G}{e^{2}}}
$$

The entire monopole, except for its Coulomb magnetic field, lies within the horizon. The exterior solution is then of the Reissner-Nordstrom form with the mass $M$ equal to the critical value for unit magnetic charge. Since we want it to be nonsingular, the interior solution cannot be simply Reissner-Nordstrom. Instead, it is similar in form to the solutions for subcritical $v$ at small $r$, while near the horizon $u$ and $1-h$ vanish as powers of $r_{H}-r$. Two aspects of this solution may seem puzzling. First, it may seem unphysical for the entire evolution of the matter fields to take place within a finite range of $r$. However, this becomes more plausible when one notes that the physical distance from the origin is

$$
l(r)=\int_{0}^{r} d r \sqrt{A(r)}
$$

Since $A$ diverges as $\left(r-r_{H}\right)^{-2}$ near the horizon of the critical solution, $l\left(r_{H}\right)$ is in fact infinite. In a sense, rather than the monopole being compressed to fit within the horizon, the horizon has been expanded outward to encompass the monopole. Second, the values for the fields and their derivatives at any $r>r_{H}$ do not determine the solution everywhere, as evidenced by the fact that this solution and the Reissner-Nordstrom agree in the exterior region but differ in the interior. This is possible because the simultaneous vanishing of $1 / A$ and $(1 / A)^{\prime}$ at the horizon prevents one from simply integrating across the horizon and allows nonanalytic behavior at $r=r_{H}$.

We have checked these arguments by numerically solving the field equations. Starting with the small distance expansions of Eqs. (3.1) and (3.2), we varied the constants $C_{u}$ and $C_{h}$ until the proper asymptotic behavior was obtained. In all cases we found that as $v$ approached $v_{c r}$ the solution tended toward one which was 
purely Reissner-Nordstrom in the exterior region, rather than one for which the fields were given by $\hat{u}$ and $\hat{h}$ at the horizon.

A sample of these results is displayed in Fig. 1, where we show $u, h$, and $1 / A$ as functions of $r$ for $\lambda / e^{2}=1.0$ and $\mu=8 \pi G v^{2}$ equal to $0.1,1.0,2.0$, and 2.35. The last of these values is as close as we were able to come to the critical value $\mu_{c r}=8 \pi G v_{c r}^{2}$. As $\mu$ increases, the monopole appears to be pulled inward. The minimum of $1 / A$ also moves inward, although less so. A contrasting view is obtained by plotting these fields as functions of the physical distance $l(r)$. As an example, $u(r)$ is plotted in this fashion in Fig. 2; we see that the change in the physical size of the monopole is actually rather small.

We also studied the behavior of $\mu_{c r}$, finding it to be a decreasing function of $\lambda / e^{2}$. In particular, for $\lambda / e^{2}$ equal to $0.1,1.0$, and $10.0, \mu_{c r}$ is $3.7,2.4$, and 1.6, respectively.

\section{Black Holes in Monopoles}

It was argued in the previous section that for $v>v_{c r}$ all solutions will have singularities. In this section we consider these supercritical solutions as well as another class of singular solutions which may be viewed as black holes embedded inside monopoles. Let us suppose that $\mathcal{M}(0)$ is nonzero and positive, with $2 G \mathcal{M}(0)$ much smaller than the monopole radius, and that $v \ll M_{P}$, so that the monopole would not by itself become a black hole. At small $r$, the effects of the matter fields can be neglected and the metric will be similar to that of a Schwarzschild black hole with mass $\mathcal{M}(0)$. At larger $r$, the gravitational effects will be small and the matter fields will resemble those of a flat space monopole. One might object that having structure outside the horizon would be forbidden by the no-hair theorems, and that the monopole would collapse into a Reissner-Nordstrom black hole. This is not so. The behavior of the fields at the outer edges of the monopole core is determined largely by the shape of the position-dependent field potential $U$ at that radius. The effects on the fields in this region of a small black hole near the center 
of the monopole would be small, much as the effects of a small black hole at the center of a large solid body (e.g., the Earth) would be neglible at the outer regions of the body. To understand how equilibrium is possible at the horizon, note that at $r=r_{H}$ the covariant conservation of a diagonal energy-momentum tensor reduces to the condition $\rho+p_{r}=0$, where $T_{\nu}^{\mu} \equiv \operatorname{diag}\left(-\rho, p_{r}, p_{\theta}, p_{\phi}\right)$. While this cannot be achieved in normal fluids, which have positive pressure, it is quite possible in a field theory.

Let us now try to make these arguments more quantitative. We begin by recalling the derivation of a no-hair theorem ${ }^{[9]}$ for a theory with a single scalar field $\phi$. We restrict our consideration to spherically symmetric configurations, so the matter field equation can be written as

$$
\left(\frac{r^{2} \sqrt{A B} \phi^{\prime}}{A}\right)^{\prime}=r^{2} \sqrt{A B} \frac{d V}{d \phi}
$$

with the metric given by Eq. (2.5). Multiplying both sides by $\left(\phi-\phi_{0}\right)$, where $\phi_{0}$ is a minimum of the $V(\phi)$, and integrating from the horizon out to infinity gives

$$
\int_{r_{H}}^{\infty} d r r^{2} \sqrt{A B}\left[\frac{1}{A} \phi^{\prime 2}+\left(\phi-\phi_{0}\right) \frac{d V}{d \phi}\right]=\left.\frac{r^{2} \sqrt{A B} \phi^{\prime}\left(\phi-\phi_{0}\right)}{A}\right|_{r=r_{H}} ^{r=\infty}
$$

The right hand side vanishes, since $1 / A\left(r_{H}\right)=0$, while energetic arguments require that $\phi^{\prime}\left(\phi-\phi_{0}\right)$ fall faster than $r^{-2}$ at large distances. The first term in the integral on the left hand side can never be negative, since $A(r)>0$ outside the horizon. If $\phi_{0}$ is the only minimum of $V(\phi)$, then the second term in the integrand is also non-negative everywhere, and Eq. (4.2) can only be satisfied if $\phi(r)=\phi_{0}$ for all $r>r_{H}$. Thus a necessary condition for the existence of a nontrivial field outside the horizon is that $V(\phi)$ have more than one minimum.

As we have seen, the monopole problem, when restricted to spherically symmetric configurations, resembles a theory with two scalar fields and a positiondependent field potential. The fields which minimize this potential are different at 
large and small values of $r$. If $2 G \mathcal{M}(0)$ is much less than both $1 /(e v)$ and $1 /(\sqrt{\lambda v})$, we would expect the fields at the horizon to be at or near the short distance minimum, $u=1, h=0$. There would then be nontrivial behavior in the region outside the horizon as the fields evolved to the asymptotic values corresponding to the large distance minimum. On the other hand, if the horizon is located at large $r$, where $u=0, h=1$ is the only minimum of the potential, the no-hair theorem derived above suggests that the fields must lie at their asymptotic value everywhere outside the horizon.

This picture can be made more precise with the aid of certain inequalities which $r_{H}$ and the values of the fields must obey, if we make a few plausible assumptions. We assume that the fields vary monotonically outside the horizon, so that $u^{\prime}$ is everywhere negative and $h^{\prime}$ is everywhere positive, with $u$ and $h$ always taking values between zero and one. At the horizon, we have $(1 / A)^{\prime} \geq 0$, with equality holding only for the critical solutions discussed in the previous section. From Eqs. (2.24)-(2.26), we then have

$$
\begin{aligned}
& u^{*}\left(1-u^{* 2}\right) \geq u^{*} h^{* 2} e^{2} v^{2} r_{H}^{2} \\
& h^{*} u^{* 2} \geq h^{*}\left(1-h^{* 2}\right) \lambda v^{2} r_{H}^{2}
\end{aligned}
$$

where $u^{*} \equiv u\left(r_{H}\right)$ and $h^{*} \equiv h\left(r_{H}\right)$. Inequalities (4.3) and (4.4) translate into the following possibilities. Either $u=0, h=1$ (corresponding to the exterior solution being Reissner-Nordstrom) or

$$
\begin{gathered}
\lambda v^{2} r_{H}^{2}\left(1-h^{* 2}\right) \leq u^{* 2} \leq 1-h^{* 2} e^{2} v^{2} r_{H}^{2} \\
1-\frac{u^{* 2}}{\lambda r_{H}^{2} v^{2}} \leq h^{* 2} \leq \frac{1-u^{* 2}}{e^{2} r_{H}^{2} v^{2}}
\end{gathered}
$$

(There is one more possibility, viz. $h^{*}=0$. This can only occur if $(1 / A)^{\prime}=0$, but we have already seen that for the critical case $h^{*}=0$ leads to singularities at the 
horizon.) Since $u^{2}$ and $h^{2}$ must lie between zero and one, these inequalities require

$$
\begin{array}{ll}
\lambda v^{2} r_{H}^{2} \leq 1 & \lambda \leq e^{2} \\
e^{2} v^{2} r_{H}^{2} \leq 1 & \lambda>e^{2}
\end{array}
$$

Since $r_{H}$ is given by $2 G \mathcal{M}\left(r_{H}\right)$, it clearly increases as either $\mathcal{M}(0)$ or $v$ is increased. Thus if we increase either of these quantities, we will eventually reach a point when these inequalities can no longer be satisfied. When this happens, the only admissible solution to the inequalities (4.3) and (4.4) is $u^{*}=0, h^{*}=1$.

One could summarize these results by drawing a "phase diagram" of the solutions as a function of $\mathcal{M}(0)$ and $v$. The nonsingular solutions considered in the previous section would lie along the $\mathcal{M}(0)=0$ axis, with $v<v_{c r}$. Above this axis, and to the left of a critical line, would be the black hole solutions we have just described. To the right of this line there would be no solutions. The ReissnerNordstrom solutions would not appear on the phase diagram, because for these $\mathcal{M}(0)=-\infty$. diagram, because for these $\mathcal{M}(0)$ is infinite.

Obtaining the precise boundaries in this phase diagram would require that we return to the field equations (2.16)-(2.18) and look for numerical solutions for various values of $\mathcal{M}(0)$ and $v$. However, considerable insight can be gained by the analysis of a somewhat simplified model of a monopole. In this model the flat space monopole is composed of a core of radius $R$ with uniform energy density, with only the Coulomb magnetic field extending outside the core. The energy density is then

$$
\rho= \begin{cases}\rho_{0}, & r<R \\ \frac{1}{2 e^{2} r^{4}}, & r>R\end{cases}
$$

Integrating this to obtain the monopole mass $M_{m o n}$, and then minimizing with 
respect to $R$, gives

$$
R=\left(\frac{1}{2 e^{2} \rho_{0}}\right)^{1 / 4}
$$

and

$$
M_{\text {mon }}=\frac{8 \pi}{3 e^{2} R}
$$

with one fourth of the monopole mass lying within the core. These results are in qualitative agreement with the exact results if $R \sim 1 / e v, \rho_{0} \sim e^{2} v^{4}$, and $M_{m o n} \sim$ $v / e$.

We now use this model to calculate $\mathcal{M}(r)$ and then use the result to determine the positions of the horizons. Specifically, in the presence of gravity we define $\rho \equiv K / A+U=\mathcal{M}^{\prime} /\left(4 \pi r^{2}\right)$, and continue to model it by Eq. (4.8), with $R$ and $M_{m o n}$ as given above. This gives

$$
\frac{\mathcal{M}(r)}{r}= \begin{cases}\frac{\mathcal{M}(0)}{r}+\frac{M_{m o n} r^{2}}{4 R^{3}}, & r<R \\ \frac{\mathcal{M}(0)+M_{m o n}}{r}-\frac{3 M_{m o n} R}{4 r^{2}}, & r>R\end{cases}
$$

The behavior of this function depends on the relative magnitudes of $\mathcal{M}(0)$ and $M_{\text {mon }}$. If $\mathcal{M}(0)<M_{\text {mon }} / 2, \mathcal{M}(r) / r$ diverges at $r=0$, falls to a minimum at $r_{1}=\left(2 \mathcal{M}(0) / M_{m o n}\right)^{1 / 3} R$, and then rises to a maximum at

$$
r_{2}=\frac{3 R M_{m o n}}{2\left[\mathcal{M}(0)+M_{m o n}\right]}
$$

with

$$
\frac{\mathcal{M}\left(r_{2}\right)}{r_{2}}=\frac{e^{2}}{8 \pi}\left[\mathcal{M}(0)+M_{m o n}\right]^{2}
$$

It then decreases monotonically to zero as $r \rightarrow \infty$. The horizons occur at the 
values of $r$ such that $2 G \mathcal{M} / r=1$. One such lies at a position $r_{H}<r_{1}$ such that

$$
\frac{M_{m o n} r_{H}^{3}}{4 R^{3}}+\mathcal{M}(0)=\frac{r_{H}}{2 G}
$$

With small $v$ (and hence small $M_{m o n}$ ), the peak at $r_{2}$ is less than $1 /(2 G)$, and this is the only horizon. As $v$ is increased, with $\mathcal{M}(0)$ held fixed, the peak at $r_{2}$ rises, reaching $1 /(2 G)$ when

$$
M_{\text {mon }}+\mathcal{M}(0)=\sqrt{\frac{4 \pi}{e^{2} G}}
$$

or, equivalently,

$$
M=M_{\text {crit }}
$$

where $M=\mathcal{M}(\infty)$ and we have introduced $M_{\text {crit }}$, the critical Reissner-Nordstrom mass for unit magnetic charge. This behavior is quite analogous to that we saw for the $\mathcal{M}(0)=0$ case. Just as in that case, non-Reissner-Nordstrom solutions are not expected to exist beyond this critical point.

If instead $\mathcal{M}(0)>M_{\text {mon }} / 2, \mathcal{M}(r) / r$ decreases monotonically. Taken at face value, our formulas would always imply the existence of a horizon. However, our discussion of the no-hair theorem suggests that for a non-trivial solution to exist the horizon must lie within the monopole core, in which case it must satisfy Eq. (4.14). Requiring that this equation have a solution with $r_{H}<R$, and using Eq. (4.10), we obtain the condition

$$
M<\frac{3}{4} M_{m o n}+\frac{M_{c r i t}^{2}}{3 M_{m o n}}
$$

We can now construct the phase diagram of solutions. This is shown in Fig. 3, where we have labeled the axes by $M_{m o n}$ (which is proportional to $v$ ) and $M$; we have chosen the latter variable rather than $\mathcal{M}(0)$ in order to be able to include the Reissner-Nordstrom solutions. The line $O A$ is given by $M=M_{m o n}$, while the line $B C$ is determined by Eq. (4.17). The nonsingular monopole solutions lie along the line $O A$, with the critical solution at point $A$. In the region above and to the left 
of this line, but below the line $A B C$, are the solutions with black holes inside nontrivial monopole configurations. Reissner-Nordstrom solutions occur everywhere above the line $M=M_{\text {crit }}$. These two regions overlap to the left of $B C$; in this portion of the diagram, there are two distinct solutions with the same values for $M$ and $M_{m o n}$. Finally, since we are excluding solutions with naked singularities, there are no solutions in the region to the right of $O A$ with $M<M_{\text {crit }}$.

\section{Discussion}

We have seen that a variety of black hole solutions may be associated with the magnetic monopoles of spontaneously broken gauge theories. The ReissnerNordstrom solutions with Abelian magnetic charge have long been known; these need only a trivial modification to accomodate the Higgs field. A notable feature of these is that they require a nonzero minimum mass for any given magnetic charge. The new class of solutions we have found can have any mass down to that of the monopole, while the mass within the horizon can be arbitrarily small. Nevertheless, the black hole certainly carries unit topological charge, since the Higgs field is topologically nontrivial on the horizon. Whether or not it contains unit magnetic charge is somewhat less clearcut, since the horizon lies in a region where the asymptotic symmetry-breaking vacuum has not yet been established and where the definition of the electromagnetic field strength is ambiguous.

It is interesting to consider the evolution of these solutions as the system moves in the $M-M_{m o n}$ plane. Since $v$ is a constant of nature (although one might perhaps envision a time-dependent $v$ in a cosmological context), this motion must be along vertical lines in the phase diagram of Fig. 3. Accretion of incident external particles would increase $M$ and move the system upward. Downward motion could arise spontaneously through Hawking radiation. In particular, a pure Reissner- 
Nordstrom solution has a Hawking temperature ${ }^{[10]}$

$$
T=\frac{1}{2 \pi G} \frac{\sqrt{M^{2}-M_{c r i t}^{2}}}{\left(M+\sqrt{M^{2}-M_{c r i t}^{2}}\right)^{2}}
$$

As this black hole radiates it loses mass and increases its temperature, thus accelerating the mass loss, until it reaches a maximum temperature

$$
T_{\max }=\frac{2}{3 \sqrt{3}(4 \pi)^{3 / 2}} e M_{P}
$$

when $M=(2 / \sqrt{3}) M_{\text {crit }}$. From this point, $T$ rapidly falls, reaching zero when $M=M_{\text {crit }}$. In the usual analysis, the critical solution is thus the stable asymptotic endpoint of the Hawking process, unless the black hole has managed to discharge its magnetic charge ${ }^{[11]}$; by choosing $e$ small enough this can be suppressed. However, our results suggest that if $v<v_{c r}$ this may not be the whole story. For the solutions we found in section 4, corresponding to black holes inside monopoles, the radius of the horizon can be easily shown, using (4.17), to be larger than the horizon radius for the Reissner-Nordstrom solution of the same mass. Classically, since the area of the horizon cannot decrease, this suggests that the Reissner-Nordstrom solutions are unstable, possibly decaying to our solutions. This can indeed be shown by a perturbation analysis around the Reissner-Nordstrom solutions. ${ }^{[12]}$ There is thus the possibility of a transition from the pure Reissner-Nordstrom solution to one in which the horizon lies within the monopole core. Once this transition has occured, there is no longer any obstacle to the complete evaporation of the horizon. These possibilities await further exploration.

\section{Acknowledgements}

We thank Joshua Frieman and Hai Ren for helpful conversations. 


\section{APPENDIX A}

If $\mathcal{M}(0)=0$, any minimum of the functional

$$
M=4 \pi \int_{0}^{\infty} d r r^{2} e^{-P(r)}(K+U)+e^{-P(0)} \mathcal{M}(0)
$$

gives a nonsingular solution of the field equations. Here

$$
P(r)=\int_{r}^{\infty} d r 8 \pi G r K
$$

while $K$ and $U$ are the gradient and potential terms given by Eqs. (2.11) and (2.12). We have seen that for $v>v_{c r} \sim M_{P}$ there are no nonsingular solutions, and hence no configuration which minimizes $M$. Since $M$ is bounded from below (see Eq. (2.21)), this implies that there must be a sequence of configurations of decreasing energy which does not converge on a limiting nonsingular configuration. In this appendix we will display such a sequence.

$M$ differs from the flat space energy functional by containing the factor of $e^{-P}$. Because of this factor, a rapid variation of the fields $u$ and $h$ about some value $r=R$ leads to a suppression of the integrand in the region $r<R$. This suggests that we consider configurations of the form

$$
\begin{gathered}
u(r)= \begin{cases}0, & r>R+\frac{\Delta}{2} \\
f_{u}(r), & |r-R|<\Delta \\
1, & r<R-\frac{\Delta}{2}\end{cases} \\
h(r)= \begin{cases}1, & r>R+\frac{\Delta}{2} \\
f_{h}(r), & |r-R|<\Delta \\
0, & r<R-\frac{\Delta}{2}\end{cases}
\end{gathered}
$$

where $f_{u}(r)$ and $f_{h}(r)$ are smooth functions interpolating between the small $r$ and large $r$ values of the fields, and the limit $\Delta \rightarrow 0$ will eventually be taken. The 
large distance values $u=0, h=1$ are chosen to minimize the potential term $U$; as will become evident shortly, the precise choice of the short distance values has no effect on the final result. For configurations of this form, $K$ vanishes everywhere except in the transition region $|r-R|<\Delta$, where it is proportional to $1 / \Delta^{2}$. It follows that $P(r)=0$ for $r>R+\Delta / 2$ and is proportional to $1 / \Delta$ for $r<R-\Delta / 2$. Hence, the entire contribution to $M$ from the interior region is suppressed by a factor of the form $e^{- \text {const. } / \Delta}$. The contribution from the exterior region, which is due entirely to $U=1 /\left(2 e^{2} r^{4}\right)$, is simply $2 \pi /\left(e^{2}(R+\Delta / 2)\right)$. In the transition region, the contribution from $U$ is clearly of order $\Delta$, while that from $K$ can be estimated by writing $r^{2}=r(R+O(\Delta))$ and noting that the leading part of the integrand is then a total derivative. This gives

$$
M=\frac{R}{2 G}+\frac{2 \pi}{e^{2} R}+\cdots
$$

where the terms represented by dots are suppressed either exponentially or by powers of $\Delta$ as $\Delta \rightarrow 0$. Minimizing with respect to $R$ gives $R=\sqrt{4 \pi G / e^{2}}+\cdots$ and $M=M_{\text {crit }}+\cdots$. As $\Delta$ tends to $0, M$ approaches the critical ReissnerNordstrom mass $M_{c r i t}$, but the limiting configuration, with $\Delta=0$, is singular at $r=R$ and thus is not an acceptable solution of the field equations.

\section{APPENDIX B}

In this appendix we derive some inequalities which apply to solutions with horizons which are not necessarily Reissner-Nordstrom outside the horizon. In particular, these apply to the solutions, considered in Sec. 4, which described black holes inside monopoles. We assume that for all $r \geq r_{H}$ the matter fields $u$ and $h$ are nonsingular and take values between 0 and 1 , that $u$ is monotonically decreasing, and that $h$ is monotonically increasing.

We first derive bounds on the mass outside the horizon. The first step is to note that, after eliminating $B$ with the aid of Eq. (2.15), Eqs. (2.17)and (2.18)for 
the matter fields can be written as

$$
\begin{aligned}
\left(\frac{u^{\prime}}{A}\right)^{\prime} & =\frac{e^{2} r^{2}}{2} \frac{\partial U}{\partial u}-\frac{8 \pi G r K u^{\prime}}{A} \\
& =\frac{u\left(u^{2}-1\right)}{r^{2}}+e^{2} u h^{2} v^{2}-\frac{8 \pi G r K u^{\prime}}{A} \\
\left(\frac{r^{2} h^{\prime}}{A}\right)^{\prime} & =\frac{r^{2}}{v^{2}} \frac{\partial U}{\partial h}-\frac{8 \pi G r^{3} K h^{\prime}}{A} \\
& =2 h u^{2}+\frac{r^{2}}{v^{2}} \frac{\partial V}{\partial h}-\frac{8 \pi G r^{3} K h^{\prime}}{A}
\end{aligned}
$$

(For brevity, we have written $V$ for the Higgs potential $\frac{\lambda}{2} v^{4}\left(h^{2}-1\right)^{2}$.) Integrating the first of these, and recalling that $u^{\prime}(\infty)=1 / A\left(r_{H}\right)=0$, we get

$$
\int_{r_{H}}^{\infty} d r\left[e^{2} v^{2} u h^{2}-\frac{8 \pi G r K u^{\prime}}{A}\right]=\int_{r_{H}}^{\infty} d r \frac{u\left(1-u^{2}\right)}{r^{2}}
$$

We now integrate Eq. (2.16) to obtain the expression

$$
M-\mathcal{M}\left(r_{H}\right)=4 \pi \int_{r_{H}}^{\infty} d r r^{2}\left(\frac{K}{A}+U\right)
$$

for the mass outside the horizon. Integrating by parts the $u^{\prime 2}$ and $h^{\prime 2}$ terms in $K$ and using the field equations (B.1) and (B.2), we obtain

$$
\begin{aligned}
M-\mathcal{M}\left(r_{H}\right)=4 & \pi \int_{r_{H}}^{\infty} d r\left[\frac{1-u^{4}}{2 e^{2} r^{2}}+r^{2} V+r^{2} \frac{h}{2}\left(-\frac{\partial V}{\partial h}+\frac{8 \pi G r K h^{\prime}}{A}\right)\right. \\
& \left.-u\left(u h^{2} v^{2}-\frac{8 \pi G r K u^{\prime}}{e^{2} A}\right)\right]
\end{aligned}
$$

Since $u(r) \leq u\left(r_{H}\right) \equiv u^{*}$ for $r \geq r_{H}$, we have, using Eq. (B.3),

$$
\begin{aligned}
\int_{r_{H}}^{\infty} d r u\left(u h^{2} v^{2}-\frac{8 \pi G r K u^{\prime}}{e^{2} A}\right) & \leq u^{*} \int_{r_{H}}^{\infty} d r\left(u h^{2} v^{2}-\frac{8 \pi G r K u^{\prime}}{e^{2} A}\right) \\
& \leq u^{* 2} \int_{r_{H}}^{\infty} d r \frac{\left(1-u^{2}\right)}{e^{2} r^{2}}
\end{aligned}
$$


Substitution of this into Eq. (B.5)leads to

$$
\begin{aligned}
M-\mathcal{M}\left(r_{H}\right) & \geq 4 \pi \int_{r_{H}}^{\infty} d r\left[\frac{1-u^{4}-2 u^{* 2}\left(1-u^{2}\right)}{2 e^{2} r^{2}}\right] \\
& \geq 4 \pi \int_{r_{H}}^{\infty} d r \frac{1-2 u^{* 2}}{2 e^{2} r^{2}}
\end{aligned}
$$

and hence

$$
M-\mathcal{M}\left(r_{H}\right) \geq \frac{2 \pi}{e^{2} r_{H}}\left(1-2 u^{* 2}\right)
$$

To get an upper bound on $M-\mathcal{M}\left(r_{H}\right)$, we start with the identity

$$
\int_{r_{H}}^{\infty} d r \frac{d}{d r}\left(r^{3} U\right)=\int_{r_{H}}^{\infty} d r\left[r^{2} U+r \frac{\partial}{\partial r}\left(r^{2} U\right)+r^{3} u^{\prime} \frac{\partial U}{\partial u}+r^{3} h^{\prime} \frac{\partial U}{\partial h}\right]
$$

The left hand side gives only a surface term at $r=r_{H}$. (The term at $r=\infty$ vanishes.) On the right hand side, the last two terms can be rewritten with the aid of the field equations (B.1) and (B.2). After some algebra and an integration by parts this gives

$$
\begin{aligned}
\int_{r_{H}}^{\infty} d r r^{2} U & =-r_{H}^{3} U\left(r_{H}\right) \\
-\int_{r_{H}}^{\infty} d r & {\left[r \frac{\partial}{\partial r}\left(r^{2} U\right)+\frac{16 \pi G r^{4} K^{2}}{A}+2 r^{3} K\left(\frac{1}{A}\right)^{\prime}+\frac{2 r u^{\prime} u^{\prime \prime}}{e^{2} A}+\frac{r v^{2} h^{\prime}\left(r^{2} h^{\prime}\right)^{\prime}}{A}\right] } \\
\quad & =-r_{H}^{3} U\left(r_{H}\right) \\
+\int_{r_{H}}^{\infty} d r & {\left[\frac{\left(1-u^{2}\right)^{2}}{e^{2} r^{2}}-2 r^{2} V-\frac{16 \pi G r^{4} K^{2}}{A}-2 r^{3} K\left(\frac{1}{A}\right)^{\prime}-\frac{r^{2} K}{A}+\frac{2 u^{\prime 2}}{e^{2} A}\right] }
\end{aligned}
$$


Inserting this into Eq. (B.4) and dropping positive terms, we obtain

$$
M-\mathcal{M}\left(r_{H}\right) \leq 4 \pi\left\{-r_{H}^{3} U\left(r_{H}\right)+\int_{r_{H}}^{\infty} d r\left[\frac{2 u^{\prime 2}}{e^{2} A}+\frac{\left(1-u^{2}\right)^{2}}{e^{2} r^{2}}\right]\right\}
$$

We now need a bound on the $u^{\prime 2}$ term. To obtain this we multiply Eq. (B.1) by $u$ and integrate from the horizon to infinity to obtain

$$
\begin{aligned}
\int_{r_{H}}^{\infty} d r \frac{u^{\prime 2}}{A} & =\int_{r_{H}}^{\infty} d r\left[\frac{u^{2}\left(1-u^{2}\right)}{r^{2}}-e^{2} v^{2} h^{2} u^{2}+\frac{8 \pi G r K u u^{\prime}}{A}\right] \\
& \leq \int_{r_{H}}^{\infty} d r \frac{u^{2}\left(1-u^{2}\right)}{r^{2}}
\end{aligned}
$$

Hence,

$$
M-\mathcal{M}\left(r_{H}\right) \leq 4 \pi\left\{-r_{H}^{3} U\left(r_{H}\right)+\int_{r_{H}}^{\infty} d r \frac{\left(1-u^{4}\right)}{e^{2} r^{2}}\right\}
$$

Dropping the $u^{4}$ in the integrand gives the inequality

$$
M-\mathcal{M}\left(r_{H}\right) \leq \frac{2 \pi}{e^{2} r_{H}}\left(1+2 u^{* 2}-u^{* 4}\right)
$$

where $u^{*} \equiv u\left(r_{H}\right)$. The inequalities (B.8) and (B.14) can be combined as

$$
\frac{2 \pi}{e^{2} r_{H}}\left(1-2 u^{* 2}\right) \leq M-\mathcal{M}\left(r_{H}\right) \leq \frac{2 \pi}{e^{2} r_{H}}\left(1+2 u^{* 2}-u^{* 4}\right)
$$

As discussed in text, when the mass is large enough so that the inequalities (4.7) are no longer respected, we have Reissner-Nordstrom solutions. In this case $u^{*}=0$ and the inequalities (B.15) simply say that the mass outside the horizon is given by $2 \pi / e^{2} r_{H}$. For the type of solutions discussed in section 4 , for which we have a horizon, but for which the exterior region is not Reissner-Nordstrom, 
these inequalities can be useful. There are bounds we can put on the masses and horizon sizes of such solutions. From Eqs. (2.12) and (2.24) we have, since $(1 / A)^{\prime}$ is positive,

$$
r_{H}^{2} \geq \frac{4 \pi G}{e^{2}}\left(1-u^{* 2}\right)^{2}
$$

With $2 G \mathcal{M}\left(r_{H}\right)=r_{H}$, the left hand side of inequality (B.15) leads to

$$
M^{2} \geq \frac{4 \pi}{G e^{2}}\left(1-2 u^{* 2}\right)
$$

and

$$
G M-\sqrt{G^{2} M^{2}-\frac{4 \pi G}{e^{2}}\left(1-2 u^{* 2}\right)} \leq r_{H} \leq G M+\sqrt{G^{2} M^{2}-\frac{4 \pi G}{e^{2}}\left(1-2 u^{* 2}\right)}
$$

The right hand side of (B.15) does not constrain $r_{H}$ unless $M^{2} \geq \frac{4 \pi}{G e^{2}}\left(1+2 u^{* 2}-u^{* 4}\right)$, in which case we get

$$
r_{H} \leq G M-\sqrt{G^{2} M^{2}-\frac{4 \pi G}{e^{2}}\left(1+2 u^{* 2}-u^{* 4}\right)}
$$

or

$$
r_{H} \geq G M+\sqrt{G^{2} M^{2}-\frac{4 \pi G}{e^{2}}\left(1+2 u^{* 2}-u^{* 4}\right)}
$$

Once we specify the value of $u$ at the horizon, these inequalities constrain the values of masses and horizon sizes. For example, for the critical solutions with $u^{*}$ given by Eq. (2.13), inequality (B.16) gives

$$
e^{2} v^{2} r_{H}^{2} \leq 1-\frac{1}{2}\left(1-\frac{M^{2} G e^{2}}{4 \pi}\right)\left(1-\frac{e^{2}}{\lambda}\right)
$$

for $\lambda>e^{2}$. For $M^{2} \leq \frac{4 \pi}{G e^{2}}$, this is a refinement of inequality (4.7) in the text. For 
$\lambda<e^{2}$, we get

$$
M^{2} \leq \frac{4 \pi\left(e^{2}+\lambda-2 \lambda e^{2} v^{2} r_{H}^{2}\right)}{G e^{2}\left(e^{2}-\lambda\right)}
$$

which requires that

$$
\lambda v^{2} r_{H}^{2} \leq 1-\frac{1}{2}\left(1-\frac{\lambda}{e^{2}}\right)
$$

This is again a refinement of (4.7). 


\section{REFERENCES}

1. J.A.Frieman and C.T.Hill, SLAC preprint SLAC-PUB-4283 (1987).

2. J.A.Harvey and J.Liu, University of Chicago preprint EFI-91-27 (1991).

3. see for example, D.V.Galt'sov and A.A.Ershov, Phys.Lett. A138, 160 (1989); M.S.Volkov and D.V.Galt'sov, JETP Lett. 50, 346 (1989); R.Bartnik and J.Mckinnon, Phys. Rev.Lett. 61, 141 (1988).

4. P. van Nieuwenhuizen, D. Wilkinson, and M.J. Perry, Phys. Rev. D13, 778 (1976).

5. E.Bogomol'nyi, Sov.J.Nucl.Phys., 24, 449 (1975); S.Coleman, S.Parke, A.Neveu, and C. Sommerfield, Phys. Rev. D15, 544 (1977).

6. F.A.Bais and R.J.Russell, Phys. Rev. D11, 2692 (1975); Y.M.Cho and P.G.O.Freund, Phys. Rev. D12, 1588 (1975).

7. T. Kirkman and C. Zachos, Phys. Rev. D24, 999 (1981).

8. P.Hajicek, Proc. Roy. Soc. A 386, 223 (1983) and J.Phys. A 16, 1191 (1983).

9. J.D.Bekenstein, Phys. Rev. D5, 1239 (1972).

10. N.D.Birrell and P.C.W.Davies, Quantum Fields in Curved Space, Cambridge University Press (1982).

11. W.A.Hiscock, Phys.Rev.Lett. 50, 1734 (1983).

12. K.Lee, V.P.Nair and E.J. Weinberg, Columbia-Fermilab preprint CU-TP540/FERMILAB-Pub-91/326-A\&T 


\section{FIGURE CAPTIONS}

1) Plots of (a) $u(r)$, (b) $h(r)$, and (c) $1 / A(r)$ for $\lambda / e^{2}=1.0$ and $\mu=8 \pi G v^{2}$ equal to 0.1 (solid line), 1.0 (dashed-dotted line), 2.0 (dotted line), and 2.35 (dashed line).

2) Plot of $u(r)$ as a function of $l(r)$, the physical distance from the origin, for $\mu=8 \pi G v^{2}$ equal to 0.1 (solid line), 1.0 (dashed-dotted line), 2.0 (dotted line), and 2.35 (dashed line).

3) The phase diagram of solutions for the simplified monopole model discussed in the text. "R-N" refers to a Reissner-Nordstrom solution with a horizon, while "Mon" refers to the solutions with a black hole inside a nontrivial monopole configuration. 